\title{
Deep Learning Image Processing Enables 40\% Faster Spinal MR Scans Which Match or Exceed Quality of Standard of Care
}

\author{
A Prospective Multicenter Multireader Study
}

\author{
S. Bash ${ }^{1}$ B. Johnson² - W. Gibbs ${ }^{3}$ T. Zhang ${ }^{4}$ - A. Shankaranarayanan ${ }^{5} \cdot$ L. N. Tanenbaum ${ }^{1,6}$ (ID \\ Received: 10 April 2021 / Accepted: 3 November 2021 / Published online: 30 November 2021 \\ (c) The Author(s) 2021
}

\begin{abstract}
Objective This prospective multicenter multireader study evaluated the performance of $40 \%$ scan-time reduced spinal magnetic resonance imaging (MRI) reconstructed with deep learning (DL).

Methods A total of 61 patients underwent standard of care (SOC) and accelerated (FAST) spine MRI. DL was used to enhance the accelerated set (FAST-DL). Three neuroradiologists were presented with paired side-by-side datasets (666 series). Datasets were blinded and randomized in sequence and left-right display order. Image features were preference rated. Structural similarity index (SSIM) and per pixel L1 was assessed for the image sets pre and post DL-enhancement as a quantitative assessment of image integrity impact.

Results FAST-DL was qualitatively better than SOC for perceived signal-to-noise ratio (SNR) and artifacts and equivalent for other features. Quantitative SSIM was high, supporting the absence of image corruption by DL processing.

Conclusion DL enables $40 \%$ spine MRI scan time reduction while maintaining diagnostic integrity and image quality with perceived benefits in SNR and artifact reduction, suggesting potential for clinical practice utility.
\end{abstract}

Keywords MRI $\cdot$ Deep learning $\cdot$ Artificial intelligence $\cdot$ Spine $\cdot$ Imaging

Abstract Presentations RSNA 2019, ASSR 2020, ECR 2020, ASNR 2020, ASSR 2021

$\triangle$ L. N. Tanenbaum

nuromri@gmail.com, tanenbaum@radnet.com

1 RadNet-San Fernando Interventional Radiology, 1510

Cotner Ave., Los Angeles, CA 90025, USA

2 Rayus Radiology, 5775 Wayzata Blvd. Suite 190, St. Louis Park, MN 55416, USA

3 Dept. of Neuroradiology, Mayo Clinic, 5777 E. Mayo Blvd., Phoenix, AZ 85054, USA

4 Apple, One Apple Park Way, Cupertino, CA 95014, USA

5 Subtle Medical, 883 Santa Cruz Ave, Menlo Park, CA 94025, USA

6 Radnet-Lenox Hill Radiology, 755 Second Avenue, New York, NY 10017, USA

\author{
Abbreviations \\ AI Artificial intelligence \\ CNN Convolutional neural network \\ DL Deep learning \\ ML Machine learning \\ NEX Number of excitations \\ SNR Signal-to-noise ratio \\ SOC Standard of care \\ SSIM Structural similarity index measure
}

Deep learning (DL) based image enhancement techniques have gained attention in recent years [1]. DL is a subset of artificial intelligence (AI) machine learning (ML) that uses multiple processing layers to progressively extract key relevant features from the input data. DL models are based on artificial neural networks, most commonly convolutional neural networks (CNN) and variations, in which data transitions through a chain of layers of transformational nodes from input to output, simulating layers of neurons. DL based solutions leverage CNNs to process large volumes 
of data through a complex framework of decision-making nodes known for exemplary performance in image recognition applications, such as the ability to recognize and categorize image features [2]. DL algorithms are applied to an array of computer vision learning tasks in many industries.

Diagnostic imaging modalities are particularly suited to benefit with opportunities such as reduced radiation and/or contrast dose for PET [3, 4], MR [5] and CT [6]. DLbased image enhancement can boost image signal-to-noise ratio (SNR) offering the potential for reduced scan times [7], enhanced patient experience [8] and improved image center efficiency. DL-based image denoising methods have demonstrated performance advantages over traditional methods of denoising [9, 10] and may be employed to bolster quality of fast acquisition of MR examinations. Fast acquisitions are accomplished by modifying conventional imaging protocol parameters to decrease scan times while maintaining resolution (reducing excitations, raising bandwidth, increasing parallel imaging factors) at the cost of increased image noise (reduced SNR). DL algorithms are then applied to the compromised fast scan data to restore SNR while maintaining image sharpness and standard of care (SOC) image quality.

This prospective multicenter multireader study was designed to evaluate $40 \%$ scan time reduced spine MR images processed with a commercially available DL reconstruction algorithm against those obtained with routine SOC scan times. Along with subjective preference rating based on typical imaging criteria, the 3 neuroradiologists also blindly assessed the comparative integrity and consistency of the DL processed images.
To quantitatively assess the integrity of image processing by the DL algorithm, we employed a structural similarity index (SSIM) [11] to evaluate for absolute errors (anatomic or pathologic data loss or aberration), and per pixel L1 difference to evaluate for differences in signal intensity.

\section{Material and Methods}

\section{Participants}

A total of 61 consecutive patients $(45.5 \pm 17.1$ years old $)$ were prospectively recruited and consented for this multicenter, multireader, randomized case-control Institutional Review Board (IRB) approved study. Each patient (28 females, 33 males) was scheduled to have a clinically indicated MRI of the cervical, thoracic, or lumbar spine.

\section{Image Acquisition}

MR imaging consisted of 14 cervical, 9 thoracic and 88 lumbar region image sets $(n=111)$. The studies were acquired from one of 5 scanners (GE; Waukesha, WI, USA, 1.5 T HDe, 2 GE 1.5 T HDxt, Siemens; Erlangen, Germany, $3 \mathrm{~T}$ Skyra, Siemens $3 \mathrm{~T}$ Verio) at 5 imaging centers (4 New York, 1 California). A clinical practice, SOC study was performed consisting of multiple routine pulse sequences: sagittal T2 $(n=23) / \mathrm{T} 1 \quad(n=21) / \mathrm{STIR}(n=18) / \mathrm{PD}(n=12)$; and axial T2 $(n=20) / \mathrm{T} 1 \quad(n=17)$ (average sequence scan time: $171.2 \pm 66.4 \mathrm{~s}$ ) for a total of 111 sequences acquired from 61 patients. In addition, each subject underwent matched pulse sequences with an accelerated (FAST) pro-
Table 1 Protocol Parameters. Typical scanning parameters for standard-of-care (SOC) and accelerated (FAST) acquisitions at $1.5 \mathrm{~T}$

\begin{tabular}{llllllll}
\hline & Scan Time & TR & TE/TI & Slice $(\mathrm{mm})$ & Matrix Size & ETL & NEX \\
\hline Sag $T 1$ & & & & & & & \\
SOC & $2: 57$ & 1550 & 10 & 4 & $320 \times 192$ & 8 & 4 \\
FAST & $1: 32$ & 1550 & 10 & 4 & $320 \times 192$ & 8 & 2 \\
Sag T2 & & & & & & & \\
SOC & $1: 31$ & 2584 & 110 & 4 & $320 \times 224$ & 21 & 2 \\
FAST & $0: 36$ & 2584 & 110 & 4 & $320 \times 224$ & 21 & 1 \\
Sag PD & & & & & & & \\
SOC & $1: 25$ & 1367 & 35 & 4 & $320 \times 224$ & 8 & 2 \\
FAST & $0: 47$ & 1367 & 35 & 4 & $320 \times 224$ & 8 & 1 \\
Sag $S T I R$ & & & & & & & \\
SOC & $2: 01$ & 4850 & $33 / 140$ & 4 & $320 \times 224$ & 8 & 2 \\
FAST & $1: 13$ & 4850 & $33 / 140$ & 4 & $320 \times 224$ & 8 & 1 \\
Ax T2 & & & & & & & \\
SOC & $1: 38$ & 4459 & 102 & 4 & $320 \times 224$ & 32 & 3 \\
FAST & $0: 30$ & 4459 & 102 & 4 & $320 \times 224$ & 32 & 2 \\
Ax T1 & & & & & & & \\
SOC & $3: 15$ & 634 & 13 & 4 & $320 \times 224$ & 3 & 2 \\
FAST & $1: 47$ & 634 & 13 & & & 3 & 1 \\
\hline
\end{tabular}


tocol (average sequence scan time: $96.2 \pm 41.2 \mathrm{~s}$ ), for an additional 111 sequences (Table 1).

\section{Image Processing}

The DL model was trained on 1000s of MR DICOM datasets from multiple vendors and clinical sites with a variety of clinical indications and field strengths, thus experiencing a range of image quality, tissue contrasts, acquisition parameters, and patient anatomies. DICOMbased processing does not utilize proprietary raw k-space input and is thus vendor agnostic. DL processing provides structure-preserving noise reduction, and the spine model does not remove imaging artifacts or intrinsically enhance image sharpness.

The DL algorithm implements image enhancement using convolutional neural network-based filtering. Original images are enhanced by running through a cascade of filter banks, where thresholding and scaling operations are applied. Separate neural network-based filters are obtained for noise reduction. The parameters of the filters were obtained through an image-guided optimization process [12-14].

The model training process typically involves several steps:

- Initialization: initialize filters and weights with small random values (e.g., random Gaussian weights).

- Forward propagation: provide training images as input to the network, propagate them through the various operations (convolution, rectified linear unit, maximum pooling, etc.), and compute the network output.

- Error calculation: calculate the errors in the output layer (target image vs. output image). Usually a final loss function (for example, sum-of-squared-error) is used to combine the error in each pixel into a single objective value which is (ideally) minimized during model training.

- Back propagation: calculate the error loss gradients with respect to all weights in the network and use techniques like gradient descent to update all filter values/weights and parameter values to minimize the output error/loss.

- Training: repeat the previous steps with all the images in the selected training dataset (e.g., 90\% of available dataset), which is called one epoch. Usually multiple (such as 100) epochs are used in model training to optimize/minimize the error objective function (described in step \#3) until the model converges into a stable result.

DL processing of the FAST scan data set (FAST-DL) was performed on an edge positioned HIPAA compliant server-virtual machine using an FDA-cleared deep learning-CNN based, image enhancement product, SubtleMR ${ }^{\mathrm{TM}}$ (Version 1.2, Subtle Medical, Menlo Park, CA, USA) with a processing time of approximately 30 s per series. All images were reviewed on a commercial DICOM viewer.

\section{Radiologic Assessment}

Three experienced neuroradiologists ( $>17$ years of experience) were presented with 666 different image series randomized in sequential and left-right display order from SOC $(n=111)$, FAST $(n=111)$, FAST-DL $(n=111)$ as paired side-by-side datasets (SOC vs. FAST, SOC vs. FAST-DL, FAST-DL vs. FAST). Image features were preference rated on a Likert scale (1-5), with 1 indicating pronounced perceived superiority of the image on the left, 2 mildly superior on the left, 3 no difference, 4 mildly superior on the right, and 5 significantly superior on the right for (1) perceived SNR; (2) perceived spatial resolution; (3) imaging artifacts; (4) cord delineation; (5) cord/CSF contrast; (6) disc related pathology; (7) bone lesions; and (8) facet/ligamentous pathology. Additionally, all 3 readers assessed each of the paired datasets for image consistency (presence or absence of apparent loss, corruption, alteration, creation or exaggeration of observed anatomy and pathology).

\section{Statistical Analysis}

Wilcoxon rank sum tests were performed to assess the statistical significance of the difference in scores for each feature in comparative datasets (Table 2). Statistical significance of the difference in scores of a dataset feature was determined by a $p$-value $<0.05$. Mean and standard deviations for the combined reader Likert scores for each feature were also calculated.

Inter-reader agreement was assessed using the Spearman rank correlation method. The coefficient varies from -1 to 1 , with -1 indicating a perfectly negative relationship (a high rating from one neuroradiologist and low rating from another) and 1 indicating a perfectly positive relationship (Table 3).

To quantitatively assess the integrity of images processed by the DL algorithm, we compared both FAST and FASTDL images to the reference SOC image. We employed SSIM to assess for absolute errors (anatomic or pathologic data loss or aberration), and per pixel L1 difference to evaluate differences in signal intensity. In addition, while not part of the subjective analysis, SOC images were also processed with DL and subjected to SSIM measures (SOC vs. SOC-DL) as an additional method of assessing the impact of DL processing (Table 4).

\section{Results}

\section{Performance}

All 666 image sets (SOC, FAST, FAST-DL) were ranked as of diagnostic quality by each of the 3 neuroradiologists. 
Table 2 Wilcoxon rank sum test results. All readers combined. $P$-value $<0.05$ (bold) suggests statistical significance for features in one dataset with respect to its comparison

\begin{tabular}{|c|c|c|c|c|c|c|}
\hline \multirow[t]{2}{*}{ Feature } & \multicolumn{2}{|c|}{ SOC vs. FAST } & \multicolumn{2}{|c|}{ FAST-DL vs. SOC } & \multicolumn{2}{|c|}{ FAST-DL vs. FAST } \\
\hline & Mean \pm Std & $P$ value & Mean \pm Std & $P$ value & Mean \pm Std & $P$ value \\
\hline$\overline{\mathrm{SNR}}$ & $3.7 \pm 0.5$ & $<0.05$ & $3.4 \pm 0.6$ & $<0.05$ & $3.9 \pm 0.4$ & $<0.05$ \\
\hline Resolution & $3.0 \pm 0.3$ & $<0.05$ & $3.0 \pm 0.3$ & 0.41 & $3.0 \pm 0.2$ & 0.25 \\
\hline Artifacts & $3.3 \pm 0.6$ & $<0.05$ & $3.1 \pm 0.5$ & $<0.05$ & $3.1 \pm 0.3$ & $<0.05$ \\
\hline Cord delineation & $3.1 \pm 0.2$ & $<0.05$ & $3.0 \pm 0.2$ & 0.25 & $3.0 \pm 0.1$ & 0.16 \\
\hline Cord/CSF contrast & $3.1 \pm 0.3$ & $<0.05$ & $3.0 \pm 0.3$ & 0.56 & $3.0 \pm 0.1$ & 1 \\
\hline Disc pathology & $3.1 \pm 0.2$ & $<0.05$ & $3.0 \pm 0.2$ & 0.64 & $3.0 \pm 0.1$ & 0.1 \\
\hline Bone lesions & $3.1 \pm 0.3$ & $<0.05$ & $3.0 \pm 0.3$ & 0.3 & $3.0 \pm 0.1$ & 0.41 \\
\hline Facet/ligamentous pathology & $3.1 \pm 0.2$ & $<0.05$ & $3.0 \pm 0.1$ & 0.26 & $3.0 \pm 0.1$ & $<0.05$ \\
\hline
\end{tabular}

FAST-DL was statistically better than SOC for perceived SNR $(3.4 \pm 0.6, p$-value $<0.05)$ and imaging artifacts $(3.1 \pm 0.5, p$-value $<0.05)$. FAST-DL and SOC were statistically equivalent for perceived spatial resolution $(3.0 \pm 0.3)$, cord delineation $(3.0 \pm 0.2)$, cord/CSF contrast $(3.0 \pm 0.3)$, disc-related pathology $(3.0 \pm 0.2)$, bone lesions $(3.0 \pm 0.3)$ and facet/ligamentous pathology $(3.0 \pm 0.1)$ (with $p$-values $>0.24)$. SOC was better than FAST for all criteria ( $p$ value $<0.05)$. FAST-DL was better than FAST for SNR, artifacts, and facet/ligamentous pathology ( $p$-value $<0.05$ ). Wilcoxon Rank Sum test results for Likert scale analysis are collectively summarized for all 3 readers in Table 2 .

Qualitative assessment of image integrity was equivalent across the 3 datasets for all 3 blinded readers, indicating that there was no perceived loss or aberration of anatomy or pathology (Fig. 1). Multisequence imaging of SOC and FAST-DL of representative patients and acquisition times are demonstrated in Fig. 2.

Spearman rank-order correlation coefficient analysis demonstrated moderately strong inter-rater agreement between the 3 blinded neuroradiologists $(\mathrm{Rho}=0.454$ for radiologist 1 vs. 2 ; Rho $=0.527$ for radiologist 1 vs. 3 ; and Rho $=0.442$ for radiologist 2 vs. 3) (Table 3).

Quantitative assessment of image similarity using the SSIM was $0.981 \pm 0.011$ for SOC vs. SOC-DL and $0.984 \pm 0.009$ for FAST vs. FAST-DL. This supports the absence of substantial anatomic aberration by DL processing of the source series (Table 4). The per pixel L1 difference for SOC vs. FAST was $37.5 \pm 17.6$, and for

Table 3 Spearman Rank-order correlation coefficient for inter-reader agreement. The scores were averaged across the reader pairs. The results indicate moderately strong inter-reader agreement for Likert scale analysis of across all 8 quality features assessed

\begin{tabular}{llll}
\hline $\begin{array}{l}\text { Spearman } \\
\text { Rho }\end{array}$ & $\begin{array}{l}\text { Radiologist } 1 \\
\text { vs. 2 }\end{array}$ & $\begin{array}{l}\text { Radiologist } 1 \\
\text { vs. 3 }\end{array}$ & $\begin{array}{l}\text { Radiologist } 2 \\
\text { vs. 3 }\end{array}$ \\
\hline & Rho $=0.454$ & Rho $=0.527$ & Rho $=0.442$ \\
\hline
\end{tabular}

SOC vs. FAST-DL was $36.7 \pm 17.4$. The Wilcox paired test differences were found to be significant $(p<0.001)$ at $4.363 \mathrm{e}^{-12}$.

\section{Discussion}

This prospective, randomized, multicenter study assessed the ability of DL enhancement to preserve perceived MR spine image quality despite $40 \%$ scan time reduction.

Blinded assessments by 3 neuroradiologists found the overall diagnostic quality of DL-enhanced MR images statistically equivalent or subjectively better than SOC across all assessed features.

MR image quality and speed are traditionally linked by constraints over signal-to-noise ratio. Scans with higher SNR and/or spatial resolution are perceived as offering better overall image quality and greater detail but requiring longer scan times when using traditional image reconstruction techniques. DL-based models in image reconstruction can overcome the SNR/scan time relationship by applying detail-preserving denoising to accelerated sequences and restoring quality to SOC levels. In our study the DL-enhanced fast images were able to provide perceived SNR benefits over even conventional SOC imaging.

MR examinations are susceptible to image degradation from artifacts, often due to patient motion related to long scan times. Motion is a significant challenge in MRI occurring in $29 \%$ of inpatient/emergency department exami-
Table 4 Structural similarity index (SSIM) results. Quantitative assessment of image similarity using the SSIM was $0.981 \pm 0.011$ for SOC vs. SOC-DL and $0.984 \pm 0.009$ for FAST vs. FAST-DL. This supports the absence of substantial anatomic aberration by DLprocessing of the source series

Structural Similarity Index (SSIM)

\begin{tabular}{ll}
\hline SOC vs. SOC-DL & $0.981 \pm 0.011$
\end{tabular}

FAST vs. FAST-DL $\quad 0.984 \pm 0.009$ 
nations and $7 \%$ of outpatient studies [15] and can lead to the need to have to repeat portions of or even complete examinations. Andre et al. found that that $19.8 \%$ of all MRI sequences need to be repeated due to motion artifact, which extrapolates to a $\$ 592$ revenue loss per hour and $\$ 115,000$ loss annually per scanner due to motion artifact [16]. In this study, DL-enhanced images statistically exceeded SOC in artifact reduction, likely reflecting shorter scan times and reduced patient motion.

Scan time reductions inherently improve patient comfort and overall experience [8]. Up to $30 \%$ of patients reported significant anxiety, largely from claustrophobia, during an MR study [17]. The authors' internal multicenter surveys have shown that even minor reductions in examination length result in a significantly higher level of patient satisfaction [8].

In our study, we achieved a scan time reduction of approximately $40 \%$ while maintaining or exceeding routine quality. If DL-enhanced fast protocols were utilized with all MR exams, one could anticipate a proportional increase in exam-based workflow efficiency for an imaging facility. Future research could explore whether scan time reduction of this scale results in a true positive impact on profitability, e.g., the ability to scan more patients per day.

A scan time acceleration of $40 \%$ was chosen for this study based on limited clinical experience. Future research might investigate greater accelerations. Work with the brain has shown image acceleration of $60 \%$ while maintaining quantitative integrity [18]. Additional research could focus on making greater image quality practical by denoising higher resolution native acquisitions.

In this study, the SOC images serve as the standard for image preference. Our randomized blinded assessment of the imaging features is meant to reflect human visual perception of comparative image quality. A radiologist's qualitative assessment of non-inferiority is critical before a DLenhanced alternative would be considered acceptable for clinical use. On the other hand, processed images should satisfy both qualitative and quantitative measures to ensure that diagnostically relevant features are not altered, and integrity of the processed image information is maintained.

Concerns exist about DL post-processing introducing instabilities in an image, where tiny perturbations in the sampling domain have been shown to be capable of translating into noticeable artifacts on the reconstructed image [19]. This has been shown for highly contrived noise additions to $\mathrm{k}$-space data and it is unclear whether such effects occur under normal operating conditions. It is important to emphasize that the current method starts from image-based data rather than $\mathrm{k}$-space, which may be less susceptible to this effect.

However, to verify the absence of data aberration on the DL post-processed images, the quantitative metric of SSIM

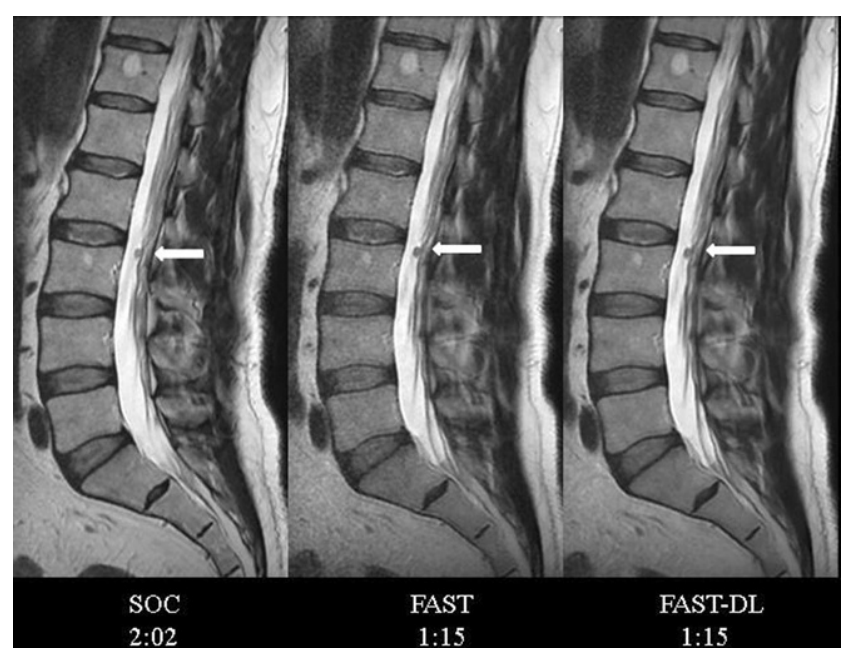

Fig. 1 Consistency across datasets. Sagittal T2 (left to right): SOC, FAST, FAST-DL with acquisition times. Blinded readers found no variations in image integrity (morphology/pathology) across the datasets. A tiny incidental intrathecal schwannoma (white arrow) at upper L3 level maintains excellent visual conspicuity across all three datasets

[11] was calculated to assess for the presence or absence of absolute errors (such as anatomic data loss or exaggeration) for the pairs of accelerated unenhanced and DL-enhanced datasets (FAST vs. FAST-DL), and as an additional measure, for the SOC series and one processed with DL solely for this purpose (SOC vs. SOC-DL). While SSIM has limitations [20], it is a commonly employed metric to measure the similarity between two images, ranging from 0.0 to 1.0 , with 1.0 meaning two images are identical. The high SSIM results for FAST vs. FAST-DL and SOC vs. SOC-DL are reassuring with respect to the absence of significant DLprocessing related corruption. As the SOC and FAST scans represent two separate acquisitions with minor differences in patient and slice position, SSIM for these could not be accurately assessed. As an additional quantitative assessment of image similarity, L1 measures were obtained. The quantitative result for image integrity is consistent with the blinded qualitative assessment by the 3 neuroradiologists who reported no instances of observed image aberration between dataset pairs (Fig. 1).

While there are numerous AI-centric solutions in the medical imaging marketspace, many have narrow application. The broad benefits of a DL solution for cross-sectional image reconstruction have been recognized, and at present MR and CT manufacturers are developing or refining DL solutions for image processing, currently at variable stages of fruition and regulatory clearance [21, 22]. Scanner vendors will likely limit their proprietary DL solutions to their own devices, and at least initially, to their newest high-end scanners [20, 21]. Independent or third-party DL solutions are vendor-agnostic and model-neutral, increasing appeal 
a

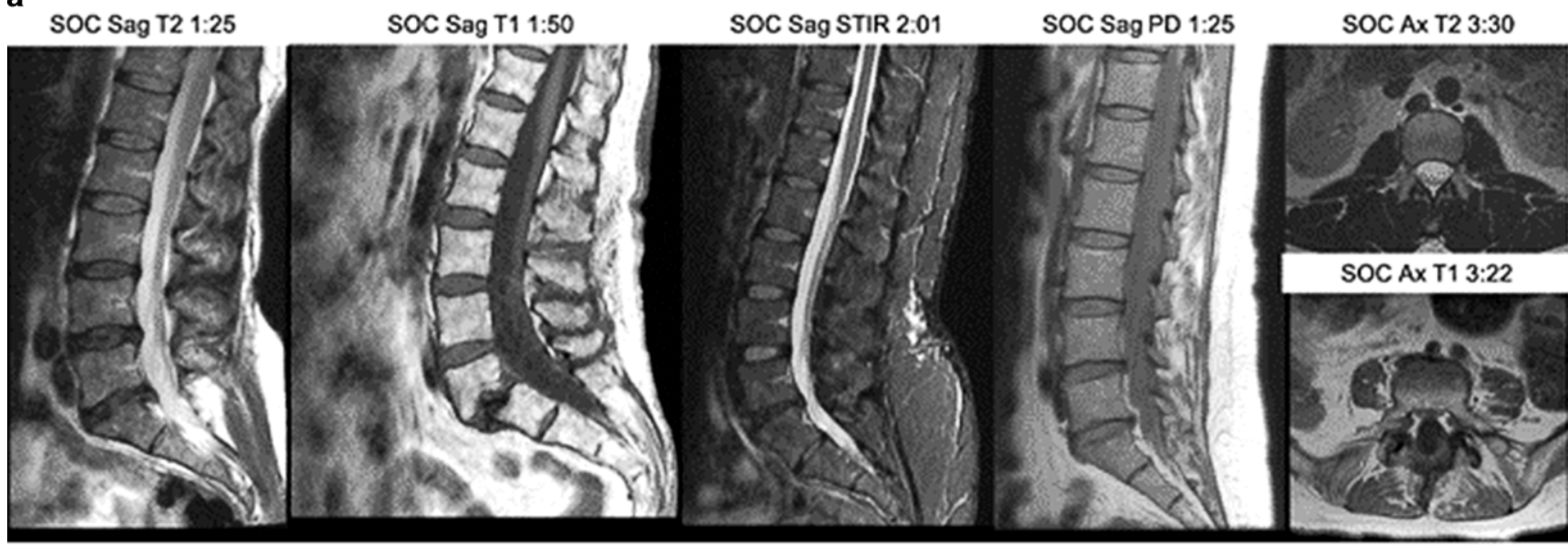

b

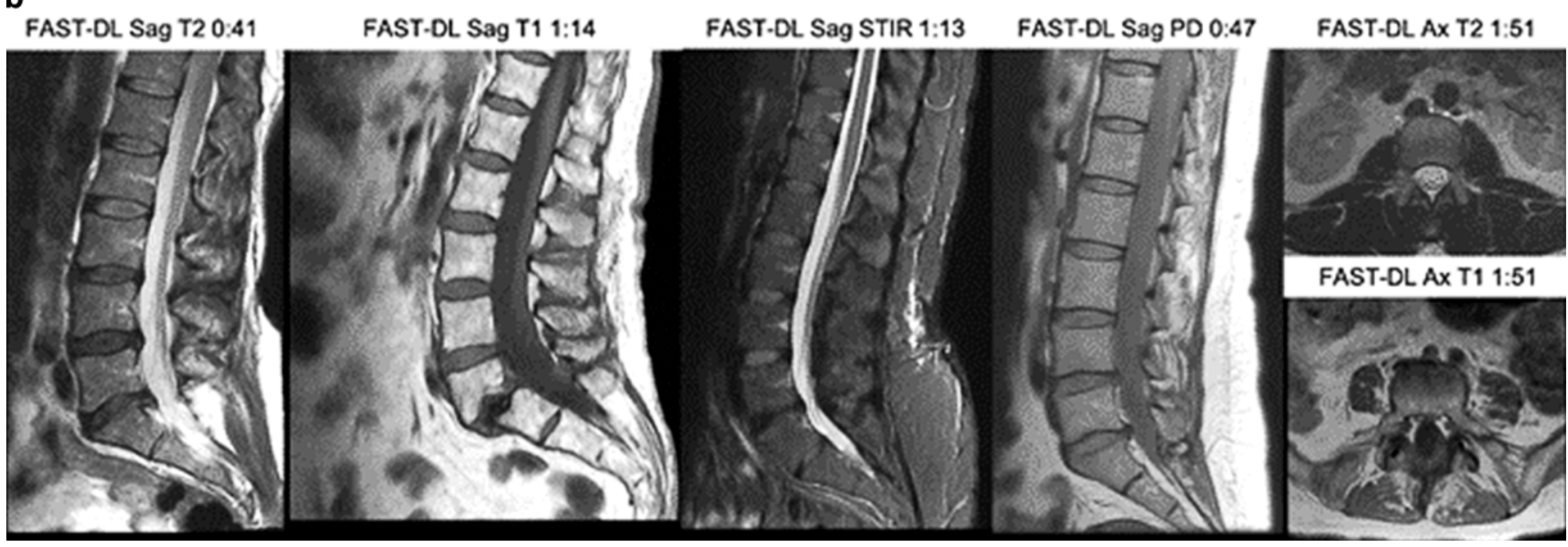

Fig. 2 Multisequence imaging. SOC (a) and FAST-DL (b) Representative patients and acquisition times

to imaging enterprises operating scanners with a variety of vendors, models, and ages.

The generalizability of our findings could be strengthened by further investigations and larger subject populations, given the relatively small number of uncommon pathologies within this study cohort. Of note, only a single intradural lesion was present and no intramedullary lesions were detected in this outpatient study and thus reported measures of cord delineation and cord/CSF contrast therefore serve as surrogates for evaluation of intradural pathology. Pathologies commonly present on outpatient spine MR studies, such as disc derangements, spinal canal stenosis, and facet arthropathy were well represented and faithfully preserved across all three datasets (SOC, FAST and FAST-DL).

In this study, clinical spine imaging patients were enrolled in a consecutive manner, a method which could both reduce as well as create bias. This led to a disproportionate number of lumbar spine studies with respect to cervical and thoracic exams; however, at the time of statistical analysis, the blinded Likert rating trends, such as perceived benefits in SNR and artifact reduction, were found to be equally applicable across all spine exams regardless of the anatomic target location.

Strengths of this investigation include the prospective, multicenter, multireader study design with images obtained from geographically diverse patient populations, using magnets of variable strength, age, and manufacturer. The results, despite evaluation in limited number of patients, support the feasibility and suggest the generalizability of DL enhancement to shorten clinical MR spine examinations.

\section{Conclusion}

DL matches or exceeds the perceived image quality and diagnostic qualitative performance of standard of care spine MRI exams, enabling a $40 \%$ scan time reduction. DL qualitatively outperformed standard of care in reduction of image artifacts and perceived signal-to-noise ratio. Quantitative structural similarity index metrics (SSIM) attest to image integrity preservation after DL-processing. This study sug- 
gests the potential for routine utility of DL reconstructed MRI in clinical practice.

Funding Partial financial support was received from Subtle Medical to the imaging institution (RadNet) to compensate for scanner time.

Conflict of interest S. Bash: Subtle Medical honoraria and consultant. B. Johnson: Subtle Medical honoraria. W. Gibbs: Subtle Medical honoraria. T. Zhang: Previous employee of Subtle Medical. A. Shankaranarayanan: Employee of Subtle Medical. L.N. Tanenbaum: Subtle Medical honoraria and consultant. Institution (RadNet): compensation for scanner use.

Open Access This article is licensed under a Creative Commons Attribution 4.0 International License, which permits use, sharing, adaptation, distribution and reproduction in any medium or format, as long as you give appropriate credit to the original author(s) and the source, provide a link to the Creative Commons licence, and indicate if changes were made. The images or other third party material in this article are included in the article's Creative Commons licence, unless indicated otherwise in a credit line to the material. If material is not included in the article's Creative Commons licence and your intended use is not permitted by statutory regulation or exceeds the permitted use, you will need to obtain permission directly from the copyright holder. To view a copy of this licence, visit http://creativecommons.org/licenses/by/4. $0 /$.

\section{References}

1. Tian C, Xu Y, Li Z, Zuo W, Fei L, Liu H. Attention-guided CNN for image denoising. Neural Netw. 2020;124:117-29.

2. Radlak K, Malinski L, Smolka B. Deep Learning Based Switching Filter for Impulsive Noise Removal in Color Images. Sensors (Basel). 2020;20:2782.

3. Kaplan S, Zhu YM. Full-Dose PET Image Estimation from LowDose PET Image Using Deep Learning: a Pilot Study. J Digit Imaging. 2019;32:773-8.

4. Xu J, Gong E, Pauly J, et al. 200x low-dose PET reconstruction using deep learning. ArXiv abs/1712.04119. 2017.

5. Gong E, Pauly JM, Wintermark M, Zaharchuk G. Deep learning enables reduced gadolinium dose for contrast-enhanced brain MRI. J Magn Reson Imaging. 2018;48:330-40.

6. Nakamura Y, Higaki T, Tatsugami F, Honda Y, Narita K, Akagi M, Awai K. Possibility of Deep Learning in Medical Imaging Focusing Improvement of Computed Tomography Image Quality. J Comput Assist Tomogr. 2020;44:161-7.

7. Zaharchuk G, Gong E, Wintermark M, Rubin D, Langlotz CP. Deep Learning in Neuroradiology. AJNR Am J Neuroradiol. 2018;39:1776-84.
8. Tanenbaum L. Quality, efficiency and survival with patient centric imaging. In: Proceedings of the 7th Snowmass 2019: Hot Topics in Radiology: Advanced Applications and Artificial Intelligence. February 10-15; Snowmass. 2019.

9. Zhang K, Zuo W, Chen Y, Meng D, Zhang L. Beyond a Gaussian Denoiser: Residual Learning of Deep CNN for Image Denoising. IEEE Trans Image Process. 2017;26:3142-55.

10. Lundervold AS, Lundervold A. An overview of deep learning in medical imaging focusing on MRI. Z Med Phys. 2019;29:102-27.

11. Wang Z, Bovik AC, Sheikh HR, Simoncelli EP. Image quality assessment: from error visibility to structural similarity. IEEE Trans Image Process. 2004;13:600-12.

12. Krizhevsky A, Sutskever I, Hinton G. ImageNet classification with deep convolutional neural networks. Commun ACM. 2017;60:84-90.

13. Loffe S, Szegedy C. Batch normalization: accelerating deep network training by reducing internal covariate shift. arXiv:1502.03167. 2015.

14. Johnson J, Alahi A, Fei-Fei L. Perceptual losses for real-time style transfer and super-resolution. In: European Conference on Computer Vision. Cham: Springer; 2016. pp. 694-711.

15. Zaitsev M, Maclaren J, Herbst M. Motion artifacts in MRI: A complex problem with many partial solutions. J Magn Reson Imaging. 2015;42:887-901.

16. Andre JB, Bresnahan BW, Mossa-Basha M, Hoff MN, Smith CP, Anzai Y, Cohen WA. Toward Quantifying the Prevalence, Severity, and Cost Associated With Patient Motion During Clinical MR Examinations. J Am Coll Radiol. 2015;12:689-95.

17. Meléndez JC, McCrank E. Anxiety-related reactions associated with magnetic resonance imaging examinations. JAMA. 1993;270:745-7.

18. Bash S, Wang L, Airriess C, et al. Deep learning enables $60 \%$ accelerated volumetric brain MRI while preserving quantitative performance-A prospective, multicenter, multireader trial. In: Proceedings of European Congress of Radiology (ECR). March 3-7; Vienna. 2021.

19. Antun V, Renna F, Poon C, Adcock B, Hansen AC. On instabilities of deep learning in image reconstruction and the potential costs of AI. Proc Natl Acad Sci U S A. 2020;117:30088-95.

20. Pambrun J, Noumeir R. Limitations of the SSIM quality metric in the context of diagnostic imaging. In: Proceedings of 2015 IEEE International Conference on Image Processing (ICIP) Quebec. 2015. pp. 2960-3.

21. Noon C. Signal boost: this AI can help take magnetic resonance images to the next level. Digital Healthcare. GE Reports. 2020.

22. Gold G. Deep learning reconstruction in clinical MRI: A new realm combining ultra-high resolution and increased productivity. Canon White Paper. 2020. 